\title{
Results on Inclusive Diffraction at HERA from the ZEUS Experiment
}

\author{
Bernd Loehr* ${ }^{* \dagger}$ \\ DESY, Notkestrasse 85, D-22607 Hamburg, Germany \\ E-mail: bernd.1oehr@desy.de
}

\begin{abstract}
Preliminary results from the ZEUS experiment on inclusive diffraction in deep inelastic electronproton scattering are presented. The data have been taken during the running of HERA in the years 1999-2000, the last period when the Leading Proton Spectrometer and the Forward Plug Calorimeter were installed. Inclusive diffractive events have been identified by three methods: forward proton detection, large rapidity gaps in proton direction, and the shape of the mass distribution measured with the ZEUS detector.
\end{abstract}

DIFFRACTION 2006 - International Workshop on Diffraction in High-Energy Physics September 5-10 2006

Adamantas, Milos island, Greece

\footnotetext{
*Speaker.

On behalf of the ZEUS Collaboration.
} 


\section{Methods to select Diffractive Events}

Inclusive diffractive reactions in Deep Inelastic Scattering (DIS) of electrons(positrons) on protons at HERA are schematically shown in Figure 1.

Between the virtual photon, emitted from the electron, and the incoming proton a colourless object is exchanged. The virtual photon dissociates into the diffractive system $\mathrm{X}$. The reaction is described by four variables: $Q^{2}$, the negative four-momentum transfer at the lepton vertex; $W$, the center of mass energy of the virtual photon and incoming proton; $t$, the four momentum transfer at the proton vertex, and $M_{X}$, the mass of the hadronic system from the dissociation of the virtual photon. The proton stays either intact or dissociates into a low mass nucleonic system N. For the majority of events the particles from the system $\mathrm{N}$ escape undetected through the beamhole of the detector. For high masses of $\mathrm{N}$ some particles might be detected. The ZEUS collaboration used three methods to select inclusive diffractive events from the measured data of inclusive DIS events at HERA. They are briefly described in the following. The data are from the run period 1999-2000, the last running period in which the Leading Proton Spectrometer [2] and the Forward Plug Calorimeter [4] have been installed. From the analysis of data from the same run period with three different methods, the ZEUS collaboration tries to arrive at a consistent picture of inclusive diffraction at HERA. This presentation is a status report and the data presented here are mostly preliminary.

The LPS-Method: The Leading Proton Spectrometer (LPS) consisted of six stations of siliconstrip detectors which are located at distances between $20 \mathrm{~m}$ and $90 \mathrm{~m}$ from the ZEUS detector in the direction of the proton beam. They are positioned between the HERA magnets very close to the proton beam and form a spectrometer which measures protons with very small transverse momenta originating from reactions in the ZEUS detector. Figure 2 shows a measured spectrum of the longitudinal momentum fraction, $x_{L}$, of scattered protons w.r.t. the incoming proton momentum. Clearly visible is the diffractive peak near $x_{L}=1$. Events at lower $x_{L}$ come from proton dissociative diffractive reactions and from nondiffractive processes. The fraction of proton dissociative events under the diffractive peak near $x_{L}=1$ is less than $1 \%$ for $x_{I P}<0.01$, where $x_{I P}$ is the momentum fraction of the colourless exchange in the diffractive reaction. Contributions from the exchange of other Regge-trajectories [1] can, however, contribute to the peak near $x=1$. The detection of the diffractively scattered proton is the only method to measure the $t$-distribution in inclusive diffractive reactions: $t=-\frac{p_{T}^{2}}{x_{L}}-\frac{\left(1-x_{L}\right)^{2}}{x_{L}} \cdot M_{p}^{2}[6]$.

The LRG-Method: In diffractive reactions there is no colour flow between the scattered proton or the nucleonic system from proton dissociation and the diffractive system from the dissociation of the virtual photon. Therefore no particles are emitted in this region. In the detector, this leads to a large (pseudo)rapidity gap (LRG) between the proton direction and the minimum angle, $\Theta_{\text {min }}$, or the highest (pseudo)rapidity $\eta_{\max }=-\ln \tan \left(\Theta_{\min } / 2\right)$, at which particles are observed. Diffractive events can be identified by the presence of such a large rapidity gaps.

The $M_{X}$-method [4]: $M_{X}$ is defined as the mass from all measured particles in the detector, except the scattered electron. The shape of the $M_{X}$-distribution is different for nondiffractive and diffractive events. Nondiffractive events lead to a rapidity-plateau distribution for the produced particles. Particle emission is a statistical process which may lead to a rapidity gap. The probability to find a rapidity gap $\Delta y$ is given by Poisson statistics, $P(0)=e^{-\lambda \Delta y}$ where $\lambda$ is the height of the 
plateau. This leads to the following mass distribution for nondiffractive events:

$$
\frac{d N_{\text {nondiff }}}{d \ln M_{x}^{2}}=c \cdot e^{b \cdot \ln M_{X}^{2}} .
$$

Diffractive events show a different $M_{X}$-distribution. For not too low and not too high values of $M_{X}$ one finds experimentally [3]

$$
\frac{d N_{d i f f}}{d M_{X}^{2}} \propto \frac{1}{M_{x}^{2}} \text { from which follows } \frac{d N_{d i f f}}{d \ln M_{X}^{2}} \approx \text { const. }
$$

This can also be derived from a Triple Regge Model [5]. For the sum of nondiffractive and diffractive events one gets :

$$
\frac{d N}{d \ln M_{x}^{2}}=D+c \cdot e^{b \cdot \ln M_{x}^{2}}
$$

Figure 3 shows a measured $\ln M_{X}^{2}$-distribution. It is compared to the properly normalized distribution of the sum of MC-simulated nondiffractive and diffractive (hatched) events. Over the range $\ln M_{X}^{2} \leq \ln W^{2}-\eta_{0}$, which is indicated by two vertical lines, the above formula with $D=$ const. is fitted to the measured distribution with $\eta_{0}$ taken from data. This gives a very good fit for the nondiffractive contribution $c \cdot e^{b \cdot \ln M_{X}^{2}}$. The fitted nondiffractive contribution is subtracted statistically from the data for each $\ln M_{X}^{2}$-bin to obtain the number of diffractive events in that bin. In the analysis only bins are used in which the diffractive part is at least $50 \%$. The diffractive events selected by the $M_{X}$-method contain contributions from proton-dissociative events. This contribution was estimated in the following way. Events were selected from a kinematical region which is dominated by proton-dissociative events and show energy deposited in the detector from the proton-dissociative system $M_{N}$. A MC-simulation of proton dissociation has been tuned to describe these events. As a result, the number of proton-dissociative events with a generated mass $M_{N} \geq 2.3 \mathrm{GeV}$ can be well described by the MC-simulation. For each kinematical $\left(Q^{2}, W, M_{X}\right)$-bin the corresponding number of MC-simulated events from proton-dissociation with masses $M_{N} \geq 2.3 \mathrm{GeV}$ has been subtracted statistically from the diffractive data. The ZEUS inclusive diffractive data selected with the $M_{X}$ method therefore contain contributions from proton-dissociative events with $M_{k}<2.3 \mathrm{GeV}$.

\section{Diffractive Structure Functions}

The inclusive diffractive cross section in DIS can be expressed in terms of diffractive structure functions in the same way as the inclusive DIS cross section is expressed by the DIS structure functions. The inclusive diffractive process in DIS is described by a fourfold differential cross section:

$$
\begin{gathered}
\frac{d^{4} \sigma_{\gamma^{*} p \rightarrow X N}^{\text {diff }} d Q^{2} d t d x_{I P} d \beta}{d \beta}=\frac{2 \pi \alpha_{e m}}{\beta Q^{2}}\left[1+(1-y)^{2}\right] \cdot\left\{F_{2}^{D(4)}\left(Q^{2}, t, x_{I P}, \beta\right)-\frac{y^{2}}{1+(1-y)^{2}} \cdot F_{L}^{D(4)}\left(Q^{2}, t, x_{I P}, \beta\right)\right\}, \\
\text { with } x_{I P}=\frac{Q^{2}+M_{x}^{2}}{Q^{2}+W^{2}} \text { and } \beta=\frac{x}{x_{I P}}=\frac{Q^{2}}{Q^{2}+M_{x}^{2}} .
\end{gathered}
$$


The contribution from the longitudinal structure function $F_{L}^{D(4)}$ is small in the kinematic region of the presented analysis and is neglected. If the measurement integrates over $t$, as in the case of the LRG- and $M_{X}$-method, the cross section becomes threefold differential:

$$
\frac{d^{3} \sigma_{\gamma^{*} p \rightarrow X N}^{d i f f}}{d Q^{2} d \beta d x_{I P}}=\frac{2 \pi \alpha_{e m}}{\beta Q^{2}}\left[1+(1-y)^{2}\right] \cdot F_{2}^{D(3)}\left(\beta, x_{I P}, Q^{2}\right) .
$$

The differential cross-section as a function of $M_{X}$ is given by:

$$
\frac{1}{2 M_{X}} \frac{d \sigma_{\gamma^{*} p \rightarrow X N}^{\text {diff }}\left(M_{X}, W, Q^{2}\right)}{d M_{X}}=\frac{4 \pi^{2} \alpha_{e m}}{Q^{2}\left(Q^{2}+M_{X}^{2}\right)} x_{I P} F_{2}^{D(3)}\left(\beta, x_{I P}, Q^{2}\right) .
$$

If $F_{2}^{D(3)}\left(\beta, x_{I P}, Q^{2}\right)$ is interpreted in terms of quark densities it specifies the probability to find in a proton, which undergoes a diffractive interaction, a quark carrying the fraction $x=\beta_{X P}$ of the proton momentum.

\section{Results from the $M_{X}$-Method}

In an earlier publication, ZEUS presented results [5] on inclusive diffraction obtained with the $M_{X}$-method which covered the range $2.7 \mathrm{GeV}^{2} \leq Q^{2} \leq 55 \mathrm{GeV}^{2}$. In this contribution new preliminary results are presented for higher values for $Q^{2}$, namely $25 \mathrm{GeV}^{2} \leq Q^{2} \leq 320 \mathrm{GeV}^{2}$. The two sets of results are called FPC I and FPC II in what follows. Figures 4 and 5 show $\frac{d \sigma^{d i f f}\left(M_{X}, W, Q^{2}\right)}{d M_{X}}$ as a function of $W$ for both datasets. In the kinematical bins $Q^{2}=25 \mathrm{GeV}^{2}$ and $Q^{2}=55 \mathrm{GeV}^{2}$, where results from both datasets exist, the cross section s agree within the errors. The structure function $x_{I P} F_{2}^{D(3)}$ as function of $x_{I P}$ obtained with the $M_{X}$-method is displayed in Figure 6 for the two datasets FPCI and FPC II. In all kinematic bins, a pronounced rise of $x_{P P} F_{2}^{D(3)}$ with decreasing $x_{I P}$ is visible. Also shown is the result of a simultaneous fit to all the data using the BEKW-model [8] in a slightly modified version [5]. The BEKW-model is a dipole model which parametrizes $x_{P} F_{2}^{D(3)}$ in terms contributions from: transverse photon $\rightarrow \overline{q q}$, longitudinal photon $\rightarrow \overline{q q}$, and transverse photon $\rightarrow q \bar{q} g$.

$$
x_{I P} F_{2}^{D(3)}\left(\beta, x_{I P}, Q^{2}\right)=c_{T} \cdot F_{q \bar{q}}^{T}+c_{L} \cdot F_{q \bar{q}}^{L}+c_{g} \cdot F_{q \bar{q} g}^{T},
$$

The modified BEKW parametrization has five free parameters which have to be determined by a fit to the data: the normalizations of the three contributions, $c_{T}, c_{L}, c_{g}$, a coefficient, $n^{T, g}$, which determines the $x_{I P}$-dependence of the transverse photon and of the $\overline{q q} g$-contribution, and a coefficient, $\gamma$, which determines the $\beta$-dependence of the $\overline{q q} g$-contribution. A fit of these five parameters to the combined FPC I + II data yield $\chi^{2} / n_{D}=0.82$ taking into account the full errors. The full line in Figure 6 shows the result of the fit. Also shown are the individual contributions. For $0.2<\beta<0.9$ the $(q \bar{q})_{T}$ contribution dominates. The gluon emission term $\overline{q q} g$ gives the largest contribution for $\beta<0.15$. The longitudinal term $(\overline{q q})_{L}$ dominates for $\beta>0.9$. This behaviour can be seen even more clearly in Figure 7 where $x_{I P} F_{2}^{D(3)}$ is plotted as a function of $\beta$ together with the BEKW-fit results. In Figure 8, $x_{I P} F_{2}^{D(3)}$ is presented as a function of $Q^{2}$ for several $x_{I P}$ and $\beta$ bins together with the BEKW-fit result. Sizable scaling violations are visible. Depending on $x_{P}$ and $\beta$ bins they range from positive scaling violations over approximate constancy to negative scaling violations. 


\section{Results from the LPS-Method}

The analysis of the diffractive events selected with the Leading Proton Spectrometer (LPS) results in a fourfold differential cross section and the structure function $x_{P} F_{2}^{D(4)}$ [7]. Figure 9 shows the measured structure function $x_{I P} F_{2}^{D(4)}$ as a function of $x_{I P}$ for $Q^{2}$ values from $2.5 \mathrm{GeV}^{2}$ to $40 \mathrm{GeV}^{2}$ at $t$ values of $|t|=0.13 \mathrm{GeV}^{2}$ and $|t|=0.3 \mathrm{GeV}^{2}$. For $x_{I P}<0.01$, the structure function rises with decreasing $x_{I P}$. For higher $x_{I P}$ it starts to rise with increasing $x_{I P}$. This latter rise is attributed to contributions from Regge-exchanges. Therefore a sum of two contributions is fitted to the data according to

$$
F_{2}^{D(4)}=f_{I P}\left(x_{I P}, t\right) \cdot F_{2}^{I P}\left(\beta, Q^{2}\right)+n_{I R} \cdot f_{I R}\left(x_{I P}, t\right) \cdot F_{2}^{I R}\left(\beta, Q^{2}\right) .
$$

The first term of the sum is the contribution from diffractive reactions, or Pomeron exchange [1], and the second one from Regge-exchanges. It is assumed that each contribution factorizes into a Pomeron- or Reggeon-structure function and corresponding flux factors. The flux factors are calculated according to Regge-Theory from the Pomeron- and the pion-trajectory. The above expression is then fitted to the data yielding the solid curves in Figure 9. Recently the H1-Collaboration published measurements of the inclusive diffractive cross section with their Forward Proton Spectrometer (FPS) in the same kinematical region [9]. They calculate a reduced cross-section, defined as

$$
\sigma_{r}^{D}=F_{2}^{D}-\frac{y^{2}}{1+(1-y)^{2}} F_{L}^{D}
$$

In Figure 10, the H1- and ZEUS-results are compared in the range $2.7 \mathrm{GeV}^{2} \leq Q^{2} \leq 24 \mathrm{GeV}^{2}$. Taking into account the normalization uncertainties of ${ }_{-10}^{+12} \%$ for the $\mathrm{H} 1$ data and $\pm 10 \%$ for the ZEUS data there is good agreement between the two experiments.

\section{Results from the LRG-Method}

Inclusive diffractive data were selected by requiring the maximum pseudorapidity in an event to be $\eta_{\max }<3.0$ outside the Forward Plug Calorimeter (FPC). In addition events were rejected with detected energies of more than $1 \mathrm{GeV}$ in the FPC. The resulting diffractive structure function $x_{I P} F_{2}^{D(3)}$ is shown in Figure 11. The result of a Regge fit, performed in the same way as described in the previous section, is shown as the continuous lines. It gives a good description of the data. No rise of $x_{I P} F_{2}^{D(3)}$ coming from Regge-exchanges can be seen because the LRG data end essentially at $x_{I P}=0.01$. In Figure 12, the ratio of the LPS data to the LRG data is shown up to $Q^{2}=40 \mathrm{GeV}^{2}$. The ratio is constant and the same in all $\left(Q^{2}, \beta\right)$-bins with the value $\mathrm{LPS} / \mathrm{LRG}=0.82 \pm 0.01$ (stat. $) \pm$ 0.03 (syst.) \pm 0.08 (norm.). Since up to $x_{I P}=0.01$ the LPS data contain no contribution from proton dissociation, this is an indication that the contribution from proton dissociation in the LRG data might be about $18 \%$. One has to take into account, however, the large normalization uncertainty of about $10 \%$ of the LPS data. In Figures 12 and 13 the $x_{I P} F_{2}^{D(3)}$ values obtained with the $M_{X}$-method and the LRG-method are compared. Figure 12 shows the comparison with the published $M_{X}$ results from 1998-1999 [5] whereas in Figure 13 the new preliminary high $Q^{2}$ data from the $M_{X^{-}}$ method are compared with the LRG data. The comparisons show reasonable agreement concerning the shape of the distributions below $x_{I P}=0.01$. Above $x_{I P}=0.01$ one can expect contributions 
from Regge exchanges in the LRG data. Indications of such contributions might be seen in some kinematical bins. Overall there seems to be a small difference in normalization between the LRGand $M_{X}$-data. Finally, in Figure 14 the $Q^{2}$-dependence of $x_{I P} F_{2}^{D(3)}$ is compared between the LRG data and the $M_{X}$ data from the low $Q^{2}$ and high $Q^{2}$ datasets. There is good agreement between the different datasets in the shape of the distributions. Again, a small difference in the overall normalization seems to be visible.

\section{Summary}

In this contribution preliminary results on inclusive diffraction from the ZEUS experiment have been presented. Results have been obtained with three different methods for the determination of the inclusive diffractive structure function $x_{P P} F_{2}^{D(3)}$ from data taken during the same running period of HERA. Comparisons between the three results of ZEUS and also between the ZEUS LPS and the H1 FPS results show reasonable agreement within the quoted errors. Some minor differences between the ZEUS LRG and $M_{X}$ results still exist. The ZEUS collaboration is still working to resolve these differences and to arrive at a consistent picture of inclusive diffraction at HERA.

\section{References}

[1] P.D.B. Collins, An Introduction to Regge Theory and High Energy Physics, Cambridge University Press, Cambridge (1977).

[2] M. Derrick et al., Z. Phys. C 73 (1977) 253.

[3] K. Goulianos, Phys. Reports 101 (1983) 169; CDF Coll., F.Abe et al., Phys. Rev. D50 (1994) 5535.

[4] ZEUS Coll., M. Derrick et al., Z. Phys. C 70 (1996) 391; ZEUS Coll., J. Breitweg et al., Eur. Phys. J. C6 (1999) 43 (1999).

[5] ZEUS Coll., S. Chekanov et al., Nucl. Phys. B 713 (2005) 3.

[6] ZEUS Coll., S. Chekanov et al., Eur. Phys. J. C38 (2004) 43.

[7] ZEUS Coll., J. Breitweg et al., Eur. Phys. J. C1 (1998) 81.

[8] J. Bartels et al., Eur. Phys. J. C7 (1999) 443.

[9] H1 Coll., A. Aktas et al., DESY-06-45 and hep-ex/0606003, to be published in Eur. Phys. J. C. 

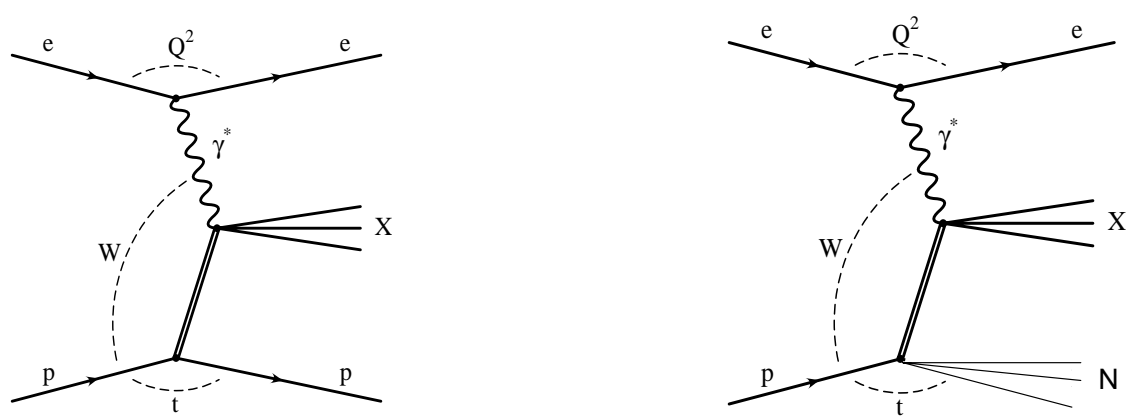

Figure 1: Schematic diagram of inclusive diffractive DIS events without and with proton dissociation.

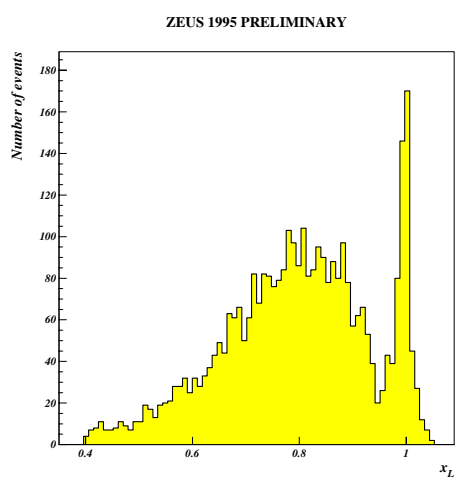

Figure 2: Fraction $x_{L}$ of the incoming proton momentum carried by the scattered proton detected in the LPS.

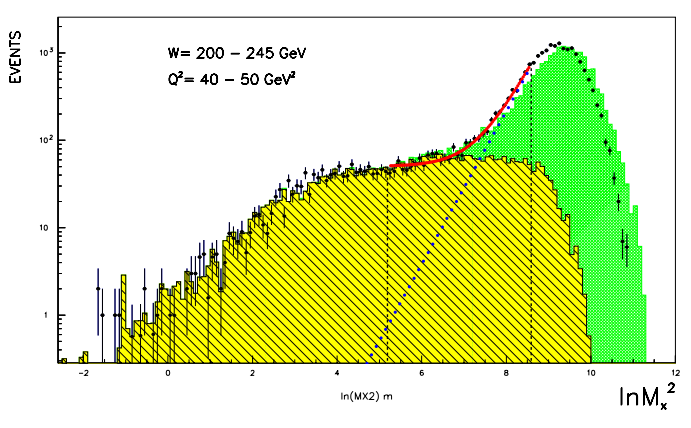

Figure 3: Measured $\ln M_{x}^{2}$ distribution. Also shown are the MC-simulated nondiffractive and diffractive (hatched) contributions. The slope of the nondiffractive contribution (dotted line) is fitted according to the formula given in the text. 


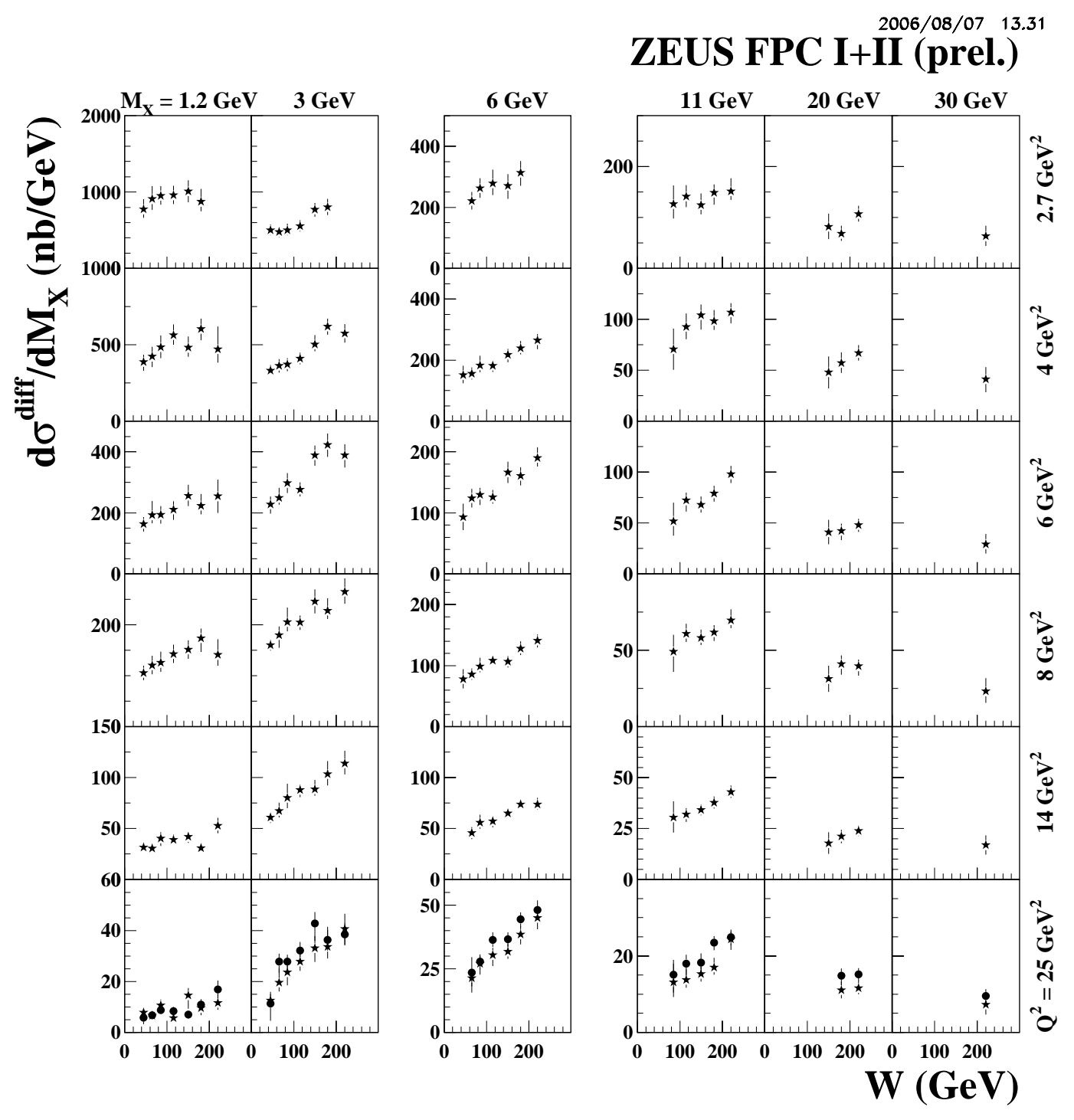

Figure 4: The differential cross sections, $d \sigma_{\gamma^{*} p \rightarrow X N}^{\text {diff }} / d M_{X}, M_{N}<2.3 \mathrm{GeV}$, as a function of $W$ for bins of $Q^{2}$ and of $M_{X}$. The inner error bars show the statistical uncertainties and the full bars the statistical and systematic uncertainties added in quadrature. Data from FPC I and II for $2.7 \leq Q^{2} \leq 25 \mathrm{GeV}^{2}$ are presented. The FPC I data are marked by stars and the FPC II data by full dots. 

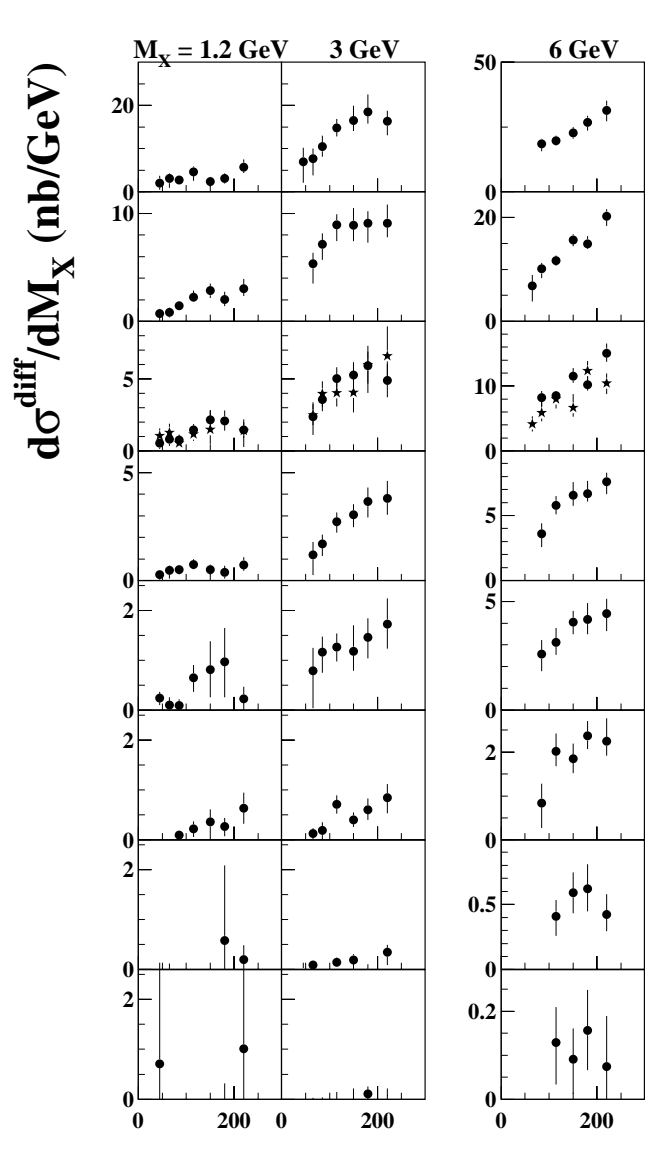

\section{ZEUS FPC I+II (prel.)}

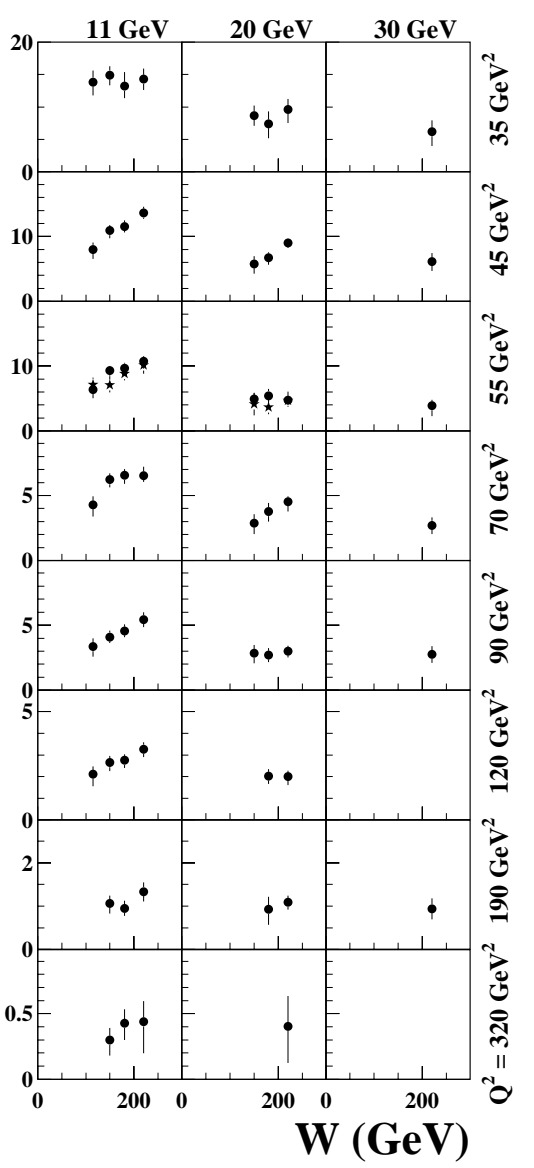

Figure 5: The differential cross sections, $d \sigma_{\gamma^{*} p \rightarrow X N}^{\text {diff }} / d M_{X}, M_{N}<2.3 \mathrm{GeV}$, as a function of $W$ for bins of $Q^{2}$ and of $M_{X}$. The inner error bars show the statistical uncertainties and the full bars the statistical and systematic uncertainties added in quadrature. Data from FPC I and II for $35 \leq Q^{2} \leq 320 \mathrm{GeV}^{2}$ are presented. The FPC I data are marked by stars and the FPC II data by full dots. 

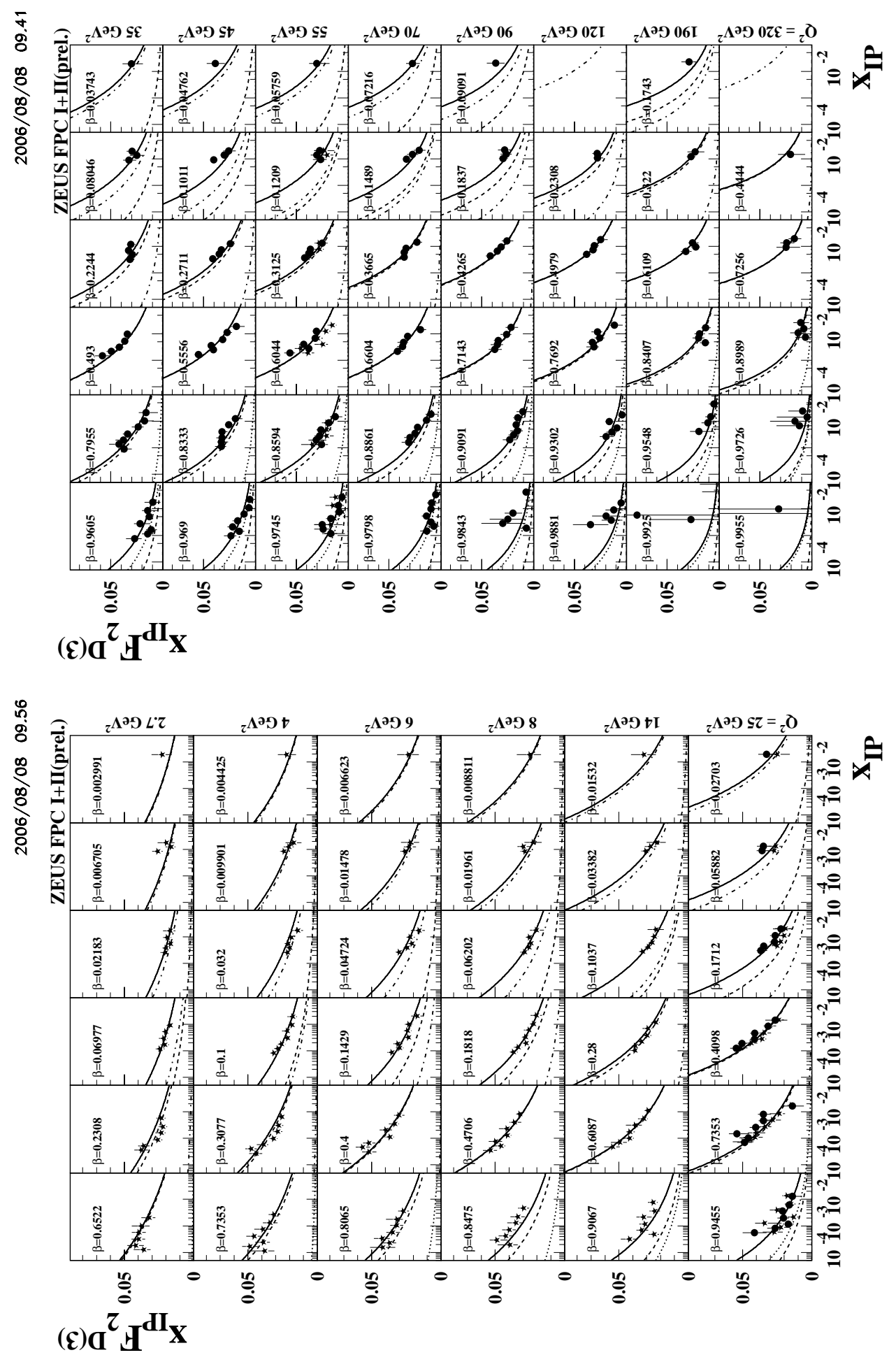

Figure 6: The diffractive structure function of the proton multiplied by $x_{I P}, x_{I P} F_{2}^{D(3)}$, for $\gamma^{*} p \rightarrow X N$, $M_{N}<2.3 \mathrm{GeV}$ as a function of $x_{I P}$ for different regions of $\beta$ : FPC I (stars) and FPC II (solid points) results are presented. The inner error bars show the statistical uncertainties and the full bars the statistical and systematic uncertainties added in quadrature. The curves show the results of the BEKW fit for the contributions from $(q \bar{q})$ for transverse (dashed) and longitudinal photons (dotted) and for the ( $q \overline{q g})$ contribution (dashed-dotted) together with the sum of all contributions (solid). 


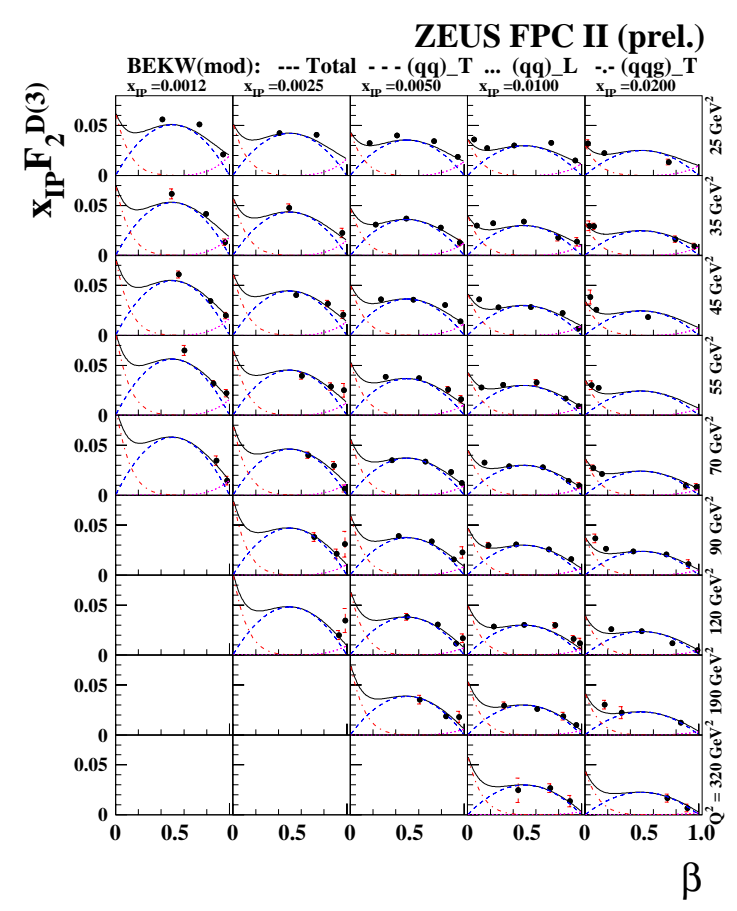

Figure 7: The diffractive structure function of the proton multiplied by $x_{I P}, x_{I P} F_{2}^{D(3)}$, as a function of $\beta$ for different regions of $Q^{2}$ and $x_{I P}$. The inner error bars show the statistical uncertainties and the full bars the statistical and systematic systematic uncertainties added in quadrature. The curves show the result of the BEKW(mod) fit for the contributions from $(q \vec{q})$ for transverse (dashed) and longitudinal photons (dotted) and for the ( $q \bar{q} g$ ) contribution (dashed-dotted) together with the sum of all contributions (solid).

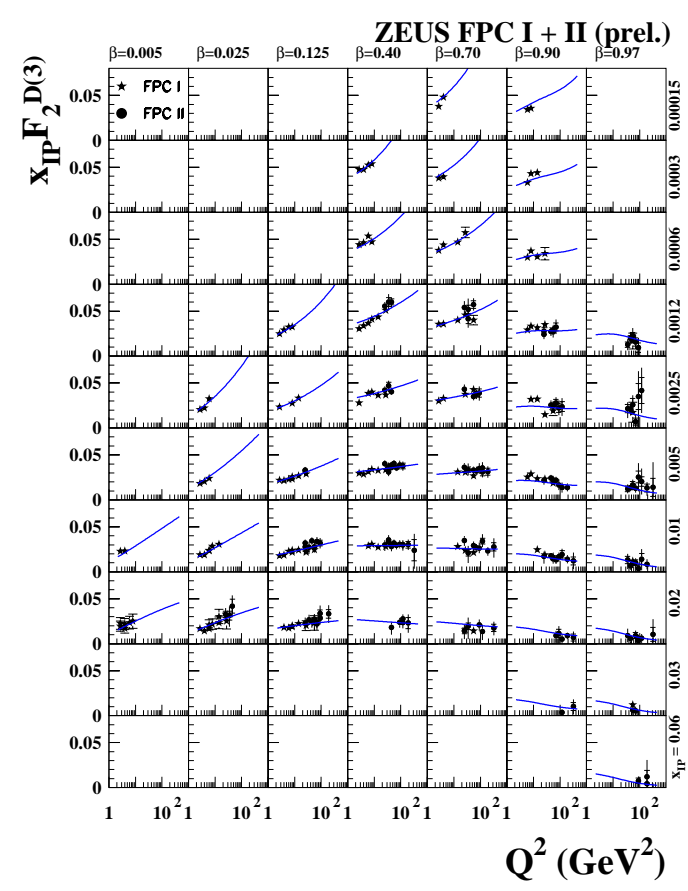

Figure 8: The diffractive structure function of the proton multiplied by $x_{I P}, x_{I P} F_{2}^{D(3)}$, as a function of $Q^{2}$ for different regions of $\beta$ and $x_{I P}$. The inner error bars show the statistical uncertainties and the full bars the statistical and systematic uncertainties added in quadrature. The curves show the result of the BEKW(mod) fit to the data of FPC I + II (solid). 


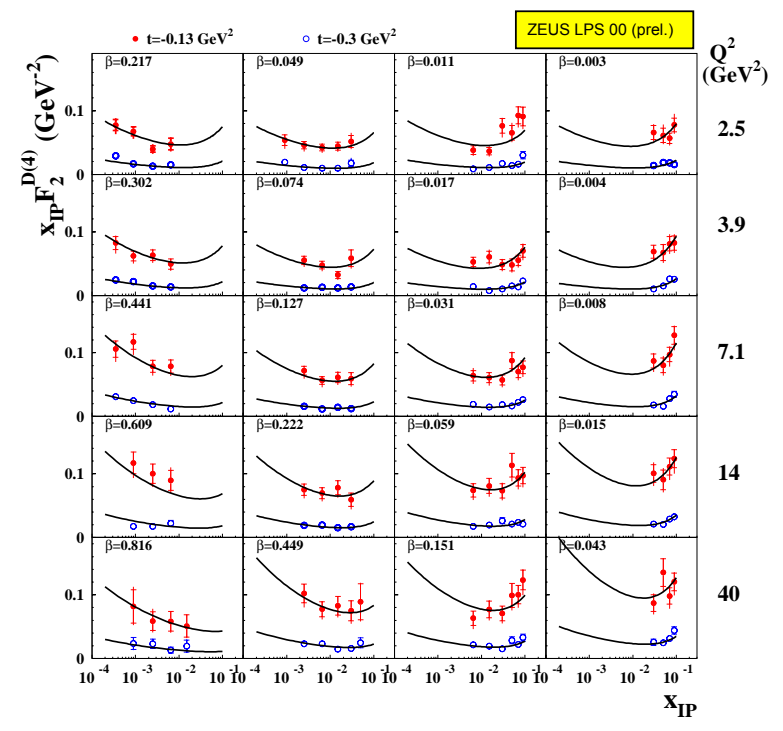

Figure 9: The structure function $X_{I P} F_{2}^{D(4)}$ in two $t$ bins as a function of $x_{I P}$ for different values of $Q^{2}$ and $\beta$. The inner error bars indicate the statistical uncertainties, the outer bars the statistical and systematic uncertainties summed in quadrature. The normalization uncertainty of $\pm 10 \%$ is not shown. The continuous lines are the result of the Regge fit described in the text.

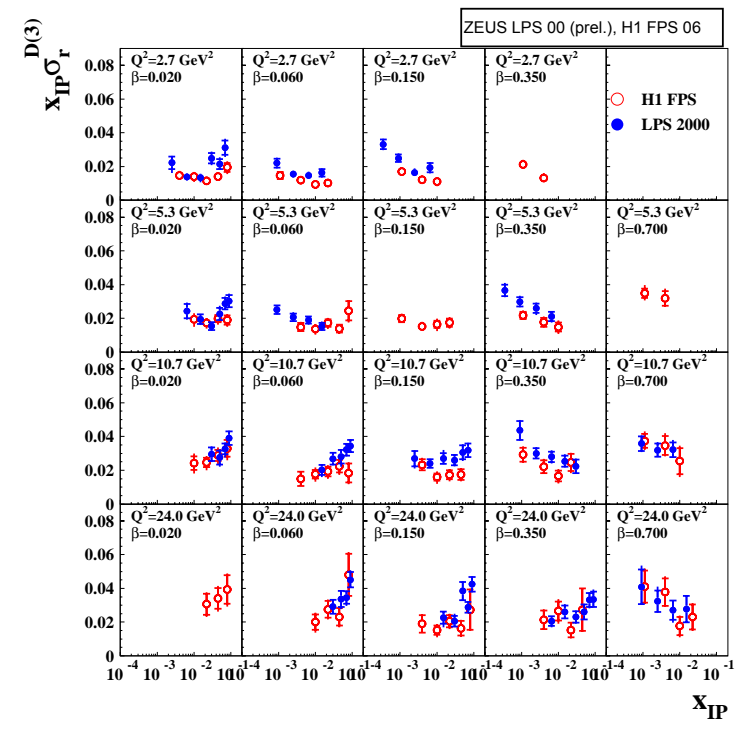

Figure 10: The reduced cross section $x_{I P} \sigma_{r}^{D(3)}$ obtained with the LPS method as a function of $x_{I P}$ for different values of $Q^{2}$ and $\beta$ compared with results obtained with the H1 FPS [9]. The inner error bars indicate the statistical uncertainties, the outer bars the statistical and systematic uncertainties summed in quadrature. The normalization uncertainty of $( \pm 10 \%)$ of the ZEUS data is not shown, nor is that of the H1 data ${ }_{-10}^{+12} \%$. 

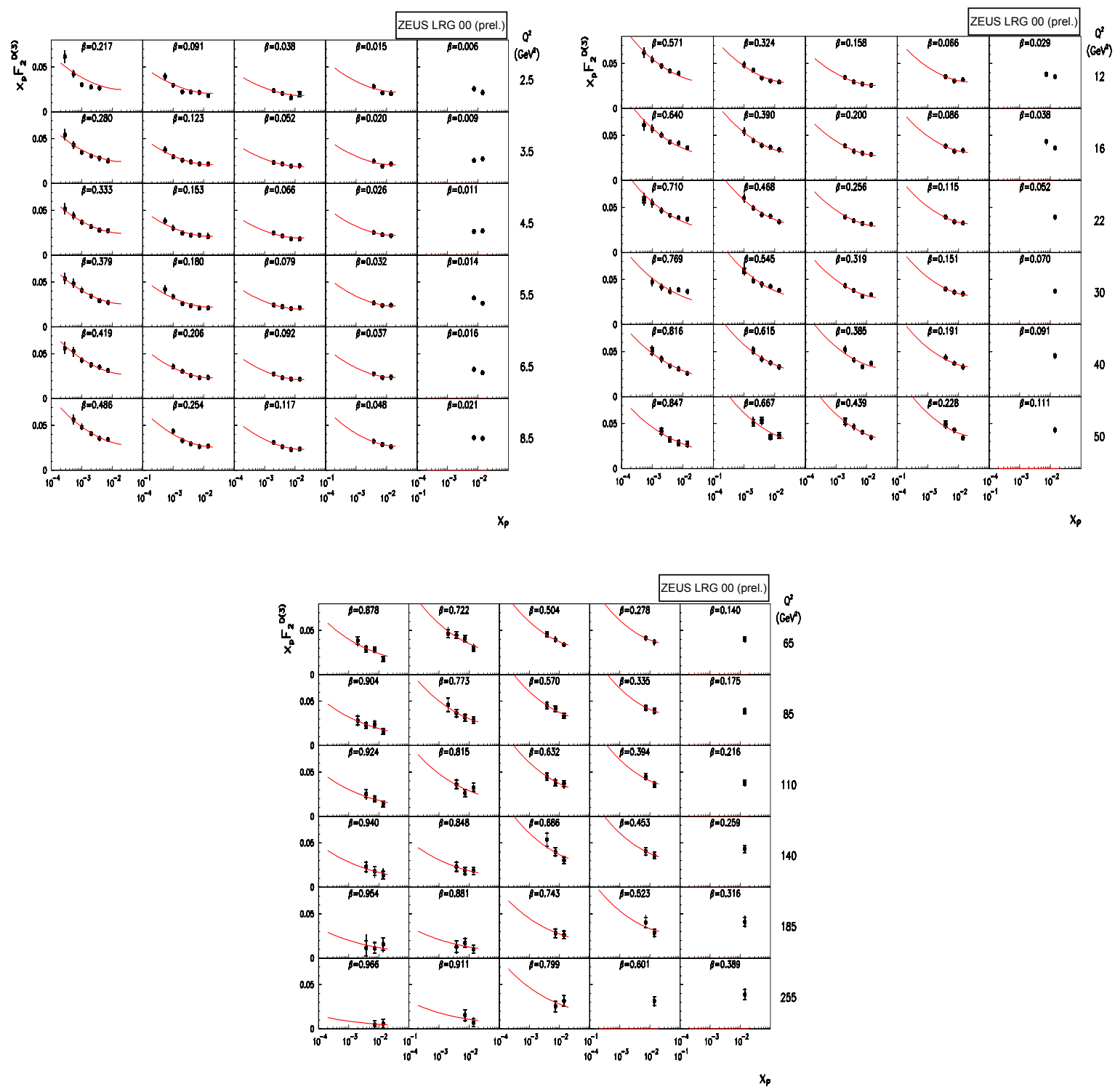

Figure 11: The structure function $x_{I P} F_{2}^{D(3)}$ obtained with the LRG method as a function of $x_{I P}$ for different values of $Q^{2}$ and $\beta$. The inner error bars indicate the statistical uncertainties, the outer bars the statistical and systematic uncertainties summed in quadrature. The normalization uncertainty of $\pm 2.25 \%$ of the ZEUS data is not shown. The continuous lines are the results of the Regge fit described in the text. 


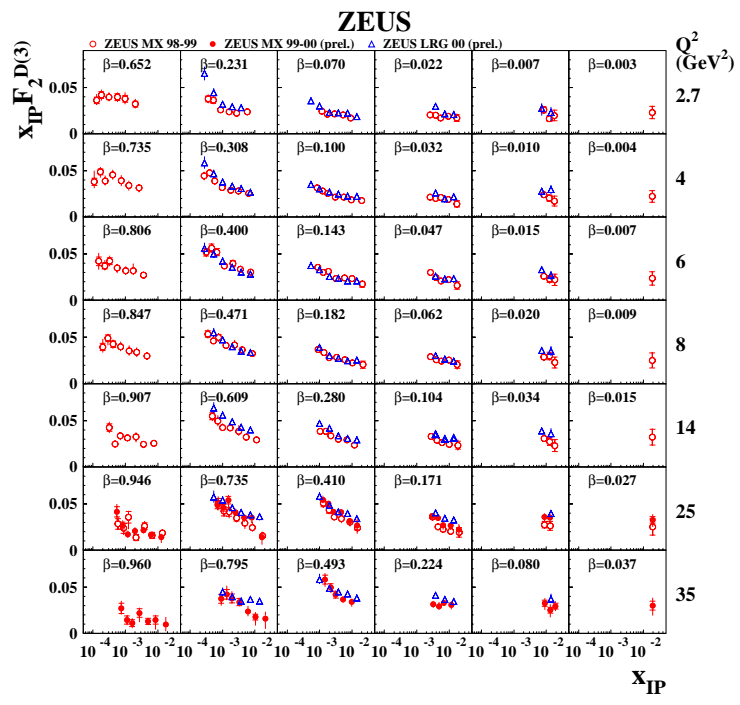

Figure 12: The structure function $x_{I P} F_{2}^{D(3)}$ obtained with the LRG method as a function of $x_{I P}$ for different values of $Q^{2}$ and $\beta$ compared with the results obtained with the $M_{X}$ method [5]. The full dots are the LRG data and the open circles are the $M_{X}$ data. The inner error bars indicate the statistical uncertainties, the outer bars the statistical and systematic uncertainties summed in quadrature.

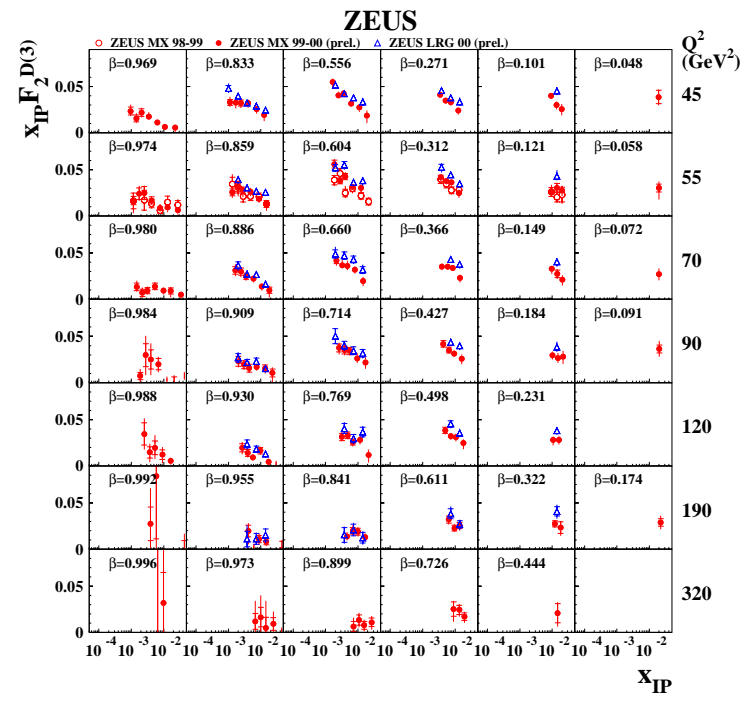

Figure 13: The structure function $x_{I P} F_{2}^{D(3)}$ obtained with the LRG method as a function of $x_{I P}$ for different values of $Q^{2}$ and $\beta$ compared with the results obtained with the $M_{X}$ method at high $Q^{2}$ from the 1999-200 data. The full dots are the LRG data and the open circles are the $M_{X}$ data. The inner error bars indicate the statistical uncertainties, the outer bars the statistical and systematic uncertainties summed in quadrature.

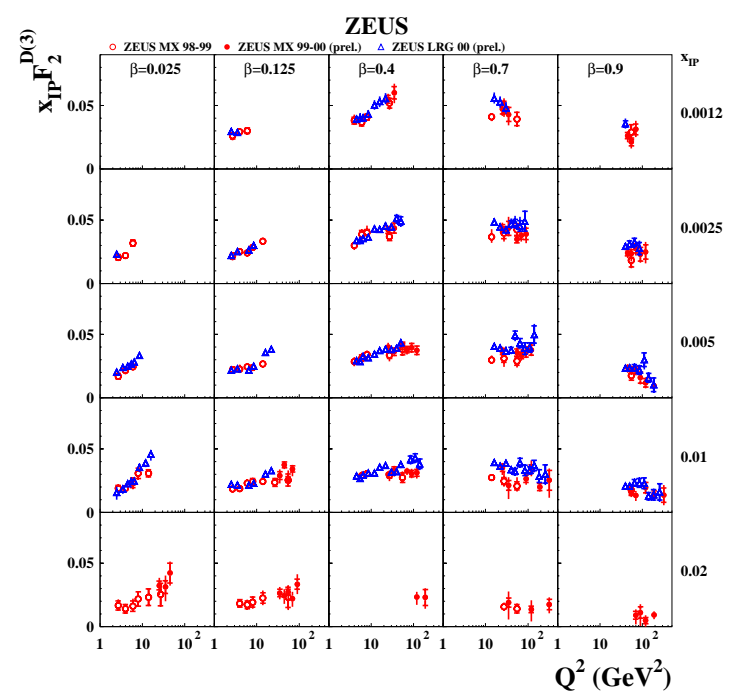

Figure 14: $x_{I P} F_{2}^{D(3)}$ is shown as a function of $Q^{2}$ for various $x_{I P}$ and $\beta$ bins. The blue points are the LRG data and the red circles and red solid points are the low $Q^{2}$ and high $Q^{2}$ data obtained with the $M_{X}$ method. 